UMDEPP 97-28

TSU-QFT-15/96

hep-th/9611193

\title{
Towards a Unified Theory of Massless Superfields of All Superspins
}

\author{
S. James Gates Jr.' \\ Department of Physics, University of Maryland \\ College Park, MD 20742-4111, USA \\ Sergei M. Kuzenkof and Alexander G. Sibiryakov? \\ Department of Quantum Field Theory, Tomsk State University \\ Lenin Ave. 36, Tomsk 634050, Russia
}

\begin{abstract}
We describe the universal linearized action for massless superfields of all superspins in $N=1, D=4$ anti-de Sitter superspace as a gauge theory of unconstrained superfields taking their values in the commutative algebra of analytic functions over a one-sheeted hyperboloid in $\mathbf{R}^{3,1}$. The action is invariant under $N=2$ supersymmetry transformations which form a closed algebra off the mass-shell.
\end{abstract}

November 1996

\footnotetext{
${ }^{1}$ E-mail: gates@umdhep.umd.edu.

${ }^{2}$ E-mail: kuzenko@phys.tsu.tomsk.su.

${ }^{3}$ E-mail: sib@phys.tsu.tomsk.su.
} 


\section{Introduction}

Off-shell superfield realizations of $N=1, D=4$ higher superspin massless multiplets were given previously [1] (see also [2]) for Poincaré supersymmetry and then extended to the case of anti-de Sitter (AdS) supersymmetry [3]. The models obtained (both with integer as well as half-integer superspins) naturally form two dually equivalent series. Any cursory glance at the structure of these models suggests that there should exist a universal formulation in which massless models of all superspins occur as special cases.

The use of such a formulation may be indispensable for constructing a consistent theory of interacting massless (super)fields of all (super)spins. In this respect it is worth mentioning a totally consistent system of equations for interacting massless fields of all spins, including the gravitational field, constructed by Vasiliev [⿴囗 ㄸ and based on a geometric generating description for (properly generalized) higher spin gauge massless models in the AdS space [5, 6]. Unfortunately, up to now it remains unknown whether these equations can be derived from an action functional.

In the present paper we propose a generating formulation (wherein each superspin enters with multiplicity one) for the combined action of free massless superfields of all superspins in $N=1, D=4$ AdS superspace $z^{M}=\left(x^{m}, \theta^{\mu}, \bar{\theta}_{\dot{\mu}}\right)$. We realize this model as a gauge theory of unconstrained superfields $\varphi\left(z^{M}, q^{a}\right)$ over the AdS superspace and analytic in a four-vector variable $q^{a}$ constrained by $q^{a} q_{a}=1$. Usual higher tensor representation superfields emerge as coefficients in power series expansions in $q$.

From a geometrical point of view, our formulation is based on enlarging the AdS superspace by a one-sheeted hyperboloid in $\mathbf{R}^{3,1}$ which is parametrized by $q^{a}$. The bosonic sector of the final superspace can be realized as a homogeneous space of $O(3,2)$, i.e. the symmetry group of the AdS space. An $O(3,2)$ covariant realization involves five-vectors $y^{\mathcal{A}}$ and $q^{\mathcal{A}}, \mathcal{A}=5,0,1,2,3$, under the constraints

$$
y^{\mathcal{A}} y_{\mathcal{A}}=-r^{2}, \quad y^{\mathcal{A}} q_{\mathcal{A}}=0, \quad q^{\mathcal{A}} q_{\mathcal{A}}=1,
$$

where the indices are contracted with the use of the metric $\eta_{\mathcal{A B}}=\operatorname{diag}(--+++)$. The constraint variables $y^{\mathcal{A}}=y^{\mathcal{A}}\left(x^{m}\right)$ parametrize the AdS space, with $-12 r^{-2}$ the corresponding curvature. A remarkable feature of the manifold introduced is that spacetime (parametrized by $y$ ) and internal space (parametrized by $q$ ) originate, in a sense, on equal footing. This becomes obvious by rewriting (1.1) as follows

$$
q_{ \pm} q_{ \pm}=0, \quad q_{+} q_{-}=1
$$

where $q_{ \pm}^{\mathcal{A}}=\left(r^{-1} y^{\mathcal{A}} \pm q^{\mathcal{A}}\right) / \sqrt{2}$. The constraints remain unchanged under $S O(1,1)$ and discrete transformations

$$
\begin{array}{rlrl}
q_{+} \rightarrow \lambda q_{+}, & & q_{-} \rightarrow \lambda^{-1} q_{-}, & \lambda \neq 0, \\
q_{+} \rightarrow q_{-}, & q_{-} \rightarrow q_{+}, &
\end{array}
$$

which commute with the AdS transformations. 
Our present formulation for the combined action of massless multiplets of all superspins is described by two real scalar superfields $X(z, q), Y(z, q)$ and a complex one $U(z, q)$, and all gauge transformations arise from a complex parameter $\varepsilon(z, q)$. The most surprising thing is that the model obtained admits a considerable simplification by imposing the algebraic gauge condition $Y=0$ which leads to an elegant formulation. What is more, the second real superfield $X$ is auxiliary and can be eliminated without explicit supersymmetry breaking. Another remarkable feature of the proposed model is that it possesses an irreducible gauge algebra instead of the infinitely reducible gauge transformations present in the original higher superspin models [3]. The approach under consideration enables us to combine all the Killing tensor superfields of the AdS superspace within a single superfield $\Upsilon(z, q)$ satisfying a simple equation. This observation presents an exceptional possibility for the covariant study of the infinite-dimensional global symmetry of the higher-superspin actions which is likely to be described by the superalgebra proposed in [7].

\section{Arbitrary Superspin Massless Models in the AdS Superspace}

The $N=1, D=4$ AdS superspace [8], with coordinates $z^{M}=\left(x^{m}, \theta^{\mu}, \bar{\theta}_{\dot{\mu}}\right)$, is specified by the algebra of covariant derivatives $\mathcal{D}_{A}=\left(\mathcal{D}_{a}, \mathcal{D}_{\alpha}, \overline{\mathcal{D}}^{\dot{\alpha}}\right)$ 円

$$
\begin{array}{lll}
\left\{\mathcal{D}_{\alpha}, \overline{\mathcal{D}}_{\dot{\alpha}}\right\}=-2 i \mathcal{D}_{\alpha \dot{\alpha}} \quad, & & {\left[\mathcal{D}_{\alpha \dot{\alpha}}, \mathcal{D}_{\beta \dot{\beta}}\right]=-2 \bar{\mu} \mu\left(\varepsilon_{\alpha \beta} \bar{M}_{\dot{\alpha} \dot{\beta}}+\varepsilon_{\dot{\alpha} \dot{\beta}} M_{\alpha \beta}\right)} \\
\left\{\mathcal{D}_{\alpha}, \mathcal{D}_{\beta}\right\}=-4 \bar{\mu} M_{\alpha \beta}, & & {\left[\mathcal{D}_{\alpha}, \mathcal{D}_{\beta \dot{\beta}}\right]=i \bar{\mu} \varepsilon_{\alpha \beta} \overline{\mathcal{D}}_{\dot{\beta}}} \\
\left\{\overline{\mathcal{D}}_{\dot{\alpha}}, \overline{\mathcal{D}}_{\dot{\beta}}\right\}=4 \mu \bar{M}_{\dot{\alpha} \dot{\beta}}, & & {\left[\overline{\mathcal{D}}_{\dot{\alpha}}, \mathcal{D}_{\beta \dot{\beta}}\right]=-i \mu \varepsilon_{\dot{\alpha} \dot{\beta}} \mathcal{D}_{\beta}}
\end{array}
$$

Here $M, \bar{M}$ denote the Lorentz generators, the non-zero constant $\mu$ of unit mass dimension determines the torsion (and hence the curvature) of the AdS superspace. The algebra of covariant derivatives is invariant under superspace general coordinate transformations and local Lorentz rotations

$$
\delta \mathcal{D}_{A}=\left[\mathcal{K}, \mathcal{D}_{A}\right], \quad \mathcal{K}=-\frac{1}{2} k^{\alpha \dot{\alpha}} \mathcal{D}_{\alpha \dot{\alpha}}+\left(k^{\alpha} \mathcal{D}_{\alpha}+k^{\alpha(2)} M_{\alpha(2)}+\text { c.c. }\right)=\overline{\mathcal{K}}
$$

\footnotetext{
${ }^{1}$ Our two-component notations and conventions coincide with those adopted in [2, 9]. We consider only Lorentz tensors symmetric in their undotted indices and separately in dotted ones. A tensor of type $(k, l)$ with $k$ undotted and $l$ dotted indices can be equivalently represented as $\psi(k, l) \equiv$ $\psi_{\alpha(k) \dot{\alpha}(l)} \equiv \psi_{\alpha_{1} \ldots \alpha_{k} \dot{\alpha}_{1} \ldots \dot{\alpha}_{l}}=\psi_{\left(\alpha_{1} \cdots \alpha_{k}\right)\left(\dot{\alpha}_{1} \cdots \dot{\alpha}_{l}\right)}$. Following Ref. [6], we assume that the indices, which are denoted by one and the same letter, should be symmetrized separately with respect to upper and lower indices; after the symmetrization, the maximal possible number of the upper and lower indices denoted by the same letter are to be contracted. In particular $\phi_{\alpha(k)} \psi_{\alpha(l)} \equiv \phi_{\left(\alpha_{1} \cdots \alpha_{k}\right.} \psi_{\left.\alpha_{k+1} \cdots \alpha_{k+l}\right)}$ and $\xi^{\alpha} \phi_{\alpha(k)} \equiv \xi^{\beta} \phi_{\left(\beta \alpha_{1} \cdots \alpha_{k-1}\right)}$. Given two tensors of the same type, their contraction is denoted by $\phi \cdot \psi \equiv \phi^{\alpha(k) \dot{\alpha}(l)} \psi_{\alpha(k) \dot{\alpha}(l)}$.
} 
with superfield parameters $k^{\alpha \dot{\alpha}}, k^{\alpha}, k^{\alpha(2)}$. The covariant derivatives remain unchanged, $\left[\mathcal{K}, \mathcal{D}_{A}\right]=0$, provided the parameters $k^{A}, k^{\alpha(2)}$ are constrained by

$$
\begin{aligned}
& k_{\alpha}=\frac{i}{8} \overline{\mathcal{D}}^{\dot{\alpha}} k_{\alpha \dot{\alpha}}, \quad k_{\alpha(2)}=\mathcal{D}_{\alpha} k_{\alpha}, \\
& \overline{\mathcal{D}}_{\dot{\alpha}} k_{\alpha \dot{\alpha}}=\mathcal{D}^{\alpha} \overline{\mathcal{D}}^{\dot{\alpha}} k_{\alpha \dot{\alpha}}=0 .
\end{aligned}
$$

Eqs. (2.2)-(2.4) define a Killing supervector $\mathcal{K}$ of the AdS superspace. The set of all Killing supervectors is known to form the superalgebra $\operatorname{osp}(1,4)$.

Now, we recall some results of [3] on the Lagrangian realization of higher-superspin massless multiplets in the AdS superspace. An important role in this approach is played by "transversal" and "longitudinal" linear superfields. A complex superfield $\Gamma(k, l)$ subject to the constraint

$$
\begin{array}{rlrl}
\overline{\mathcal{D}}^{\dot{\alpha}} \Gamma_{\alpha(k) \dot{\alpha}(l)}=0 \quad, \quad \Leftrightarrow \quad(\overline{\mathcal{P}}-l-2) \Gamma(k, l) & =0, & & l>0 ; \\
(\overline{\mathcal{P}}-2) \Gamma(k, 0) & =0, & l=0 ;
\end{array}
$$

is defined as a transversal linear superfield; a complex superfield $G(k, l)$ subject to the constraint

$$
\overline{\mathcal{D}}_{\dot{\alpha}} G_{\alpha(k) \dot{\alpha}(l)}=0 \quad, \quad \Leftrightarrow \quad(\overline{\mathcal{P}}+l) G(k, l)=0 .
$$

is defined as a longitudinal linear superfield. Here we have used the notations

$$
\mathcal{P}=\frac{1}{2 \bar{\mu}} \mathcal{D}^{2}=\frac{1}{2 \bar{\mu}} \mathcal{D}^{\alpha} \mathcal{D}_{\alpha} \quad, \quad \overline{\mathcal{P}}=\frac{1}{2 \mu} \overline{\mathcal{D}}^{2}=\frac{1}{2 \mu} \overline{\mathcal{D}}_{\dot{\alpha}} \overline{\mathcal{D}}^{\dot{\alpha}}
$$

For $l=0$ (2.6) coincides with the chirality constraint.

For each superspin value greater $3 / 2$, there are known [3] exactly two dually equivalent superfield formulations called transversal and longitudinal. In the present paper we make use of the transversal formulation for a half-integer superspin $s+1 / 2$, $s \geq 1$, and of the longitudinal formulation for an integer superspin $s, s \geq 1$. The dynamical superfield variables in these cases take the forms

$$
\begin{aligned}
\mathcal{V}_{s+\frac{1}{2}}^{\perp} & =\{H(s, s), \Gamma(s-1, s-1), \bar{\Gamma}(s-1, s-1)\}, & & s \geq 1 \\
\mathcal{V}_{s}^{\|} & =\left\{H^{\prime}(s-1, s-1), G(s, s), \bar{G}(s, s)\right\}, & & s \geq 1
\end{aligned}
$$

where $H$ and $H^{\prime}$ are real unconstrained, $\Gamma$ transversal linear and $G$ longitudinal linear superfields. The transversal superspin- $(s+1 / 2)$ action reads

$$
\begin{aligned}
S_{s+\frac{1}{2}}^{\perp}=(-1)^{s} \frac{1}{2} \int \mathrm{d}^{8} z E^{-1}\left\{\frac{1}{8} H^{\alpha(s) \dot{\alpha}(s)} \mathcal{D}^{\beta}\left(\overline{\mathcal{D}}^{2}-4 \mu\right) \mathcal{D}_{\beta} H_{\alpha(s) \dot{\alpha}(s)}\right. \\
+H^{\alpha(s) \dot{\alpha}(s)}\left(\mathcal{D}_{\alpha} \overline{\mathcal{D}}_{\dot{\alpha}} \Gamma_{\alpha(s-1) \dot{\alpha}(s-1)}-\overline{\mathcal{D}}_{\dot{\alpha}} \mathcal{D}_{\alpha} \bar{\Gamma}_{\alpha(s-1) \dot{\alpha}(s-1)}\right) \\
\left.+\frac{s^{2}}{2} \bar{\mu} \mu H \cdot H+2 \bar{\Gamma} \cdot \Gamma+\frac{s+1}{s}(\Gamma \cdot \Gamma+\bar{\Gamma} \cdot \bar{\Gamma})\right\}
\end{aligned}
$$


and the longitudinal superspin- $s$ action is given by

$$
\begin{aligned}
S_{s}^{\|}=(-1)^{s} \frac{1}{2} \int \mathrm{d}^{8} z E^{-1}\left\{\frac{1}{8} H^{\prime \alpha(s-1) \dot{\alpha}(s-1)} \mathcal{D}^{\beta}\left(\overline{\mathcal{D}}^{2}-4 \mu\right) \mathcal{D}_{\beta} H_{\alpha(s-1) \dot{\alpha}(s-1)}^{\prime}\right. \\
+\frac{s}{s+1} H^{\prime \alpha(s-1) \dot{\alpha}(s-1)}\left(\mathcal{D}^{\alpha} \overline{\mathcal{D}}^{\dot{\alpha}} G_{\alpha(s) \dot{\alpha}(s)}-\overline{\mathcal{D}}^{\dot{\alpha}} \mathcal{D}^{\alpha} \bar{G}_{\alpha(s) \dot{\alpha}(s)}\right) \\
\left.+\frac{(s+1)^{2}}{2} \bar{\mu} \mu H^{\prime} \cdot H^{\prime}+2 \bar{G} \cdot G+\frac{s}{s+1}(G \cdot G+\bar{G} \cdot \bar{G})\right\} .
\end{aligned}
$$

Here $\mathrm{d}^{8} z E^{-1}$ is the measure of the AdS superspace. Any of the models (2.10) and (2.11) describes the massless on-shell $\operatorname{osp}(1,4)$-representation of the corresponding superspin and its conjugate representation. The action $S_{s+1 / 2}^{\perp}$ is invariant under the following gauge transformations

$$
\begin{aligned}
\delta H(s, s) & =g(s, s)+\bar{g}(s, s) \\
\delta \Gamma_{\alpha(s-1) \dot{\alpha}(s-1)} & =\frac{s}{2(s+1)} \overline{\mathcal{D}}^{\dot{\alpha}} \mathcal{D}^{\alpha} \bar{g}_{\alpha(s) \dot{\alpha}(s)} .
\end{aligned}
$$

with a longitudinal linear parameter $g(s, s)$. The action $S_{s}^{\|}$is invariant under the following gauge transformations

$$
\begin{aligned}
\delta H^{\prime}(s-1, s-1) & =\gamma(s-1, s-1)+\bar{\gamma}(s-1, s-1) \\
\delta G_{\alpha(s) \dot{\alpha}(s)} & =\frac{1}{2} \overline{\mathcal{D}}_{\dot{\alpha}} \mathcal{D}_{\alpha} \bar{\gamma}_{\alpha(s-1) \dot{\alpha}(s-1)} .
\end{aligned}
$$

with a transversal linear parameter $\gamma(s, s)$. It was mentioned in [3] that the linear dynamical variables $\Gamma, G$ and their gauge parameters $g, \gamma$ can always be re-expressed via unconstrained superfields at the cost of introducing an additional gauge freedom, for both formulations (2.10) and (2.11), of infinite stage of reducibility. We are going to show that there exists a generating formulation in terms of unconstrained superfields for the unified theory of massless multiplets of all superspins with the action

$$
S=S_{0}+\sum_{s=1}^{\infty} S_{s}^{\|}+S_{\frac{1}{2}}+\sum_{s=1}^{\infty} S_{s+\frac{1}{2}}^{\perp}
$$

Here the scalar multiplet (superspin-0) is ordinarily described by a non-gauge (anti)chiral scalar superfield

$$
\mathcal{V}_{0}=\{G, \bar{G}\}, \quad \overline{\mathcal{D}}_{\dot{\alpha}} G=0
$$

with the action

$$
S_{0}=\int \mathrm{d}^{8} z E^{-1} \bar{G} G
$$

We also make use of the standard description of the vector multiplet (superspin-1/2) by a real scalar superfield

$$
\mathcal{V}_{\frac{1}{2}}=\{H\}
$$

with the action

$$
S_{\frac{1}{2}}=\frac{1}{16} \int \mathrm{d}^{8} z E^{-1} H \mathcal{D}^{\alpha}\left(\overline{\mathcal{D}}^{2}-4 \mu\right) \mathcal{D}_{\alpha} H,
$$


and the gauge invariance

$$
\delta H=g+\bar{g}, \quad \overline{\mathcal{D}}_{\dot{\alpha}} g=0 .
$$

An important observation suggesting the existence of such a formulation is based on a property of the AdS superspace that a complex tensor superfield $V(k, l)$ possess a unique decomposition into its transversal and longitudinal parts

$$
U(k, l)=\Gamma(k, l)+G(k, l) .
$$

As a consequence, one can equivalently convert the constrained dynamical variables $\Gamma(s, s)$ (from the superspin- $(s+3 / 2)$ multiplet) and $G(s, s)$ (from the superspin- $s$ multiplet) into an unconstrained complex superfield $U(s, s)$ and completely analogous for the gauge parameters $\gamma(s, s)$ and $g(s, s)$.

\section{Generating Formulation}

The system with action (2.14) can be reformulated as a gauge theory in AdS superspace with all the dynamical superfields as well as gauge parameters taking their values in the algebra of analytic functions over the one-sheeted hyperboloid in $\mathbf{R}^{3,1}$

$$
q^{a} q_{a}=-\frac{1}{2} q^{\alpha \dot{\alpha}} q_{\alpha \dot{\alpha}}=1
$$

An analytic function $\phi(q)$ is completely specified by a set of tensor coefficients $\phi_{\alpha(s) \dot{\alpha}(s)}$ (symmetric in undotted and dotted indices sepatately), $s=0,1, \ldots$, that originate in the power series

$$
\phi(q)=\sum_{s=0}^{\infty} \phi_{\alpha(s) \dot{\alpha}(s)} q^{\alpha(s) \dot{\alpha}(s)}
$$

where

$$
q^{\alpha(s) \dot{\alpha}(s)}=\underbrace{q^{\alpha \dot{\alpha}} \cdots q^{\alpha \dot{\alpha}}}_{s \text { times }} .
$$

The algebra possesses the unique, modulo normalization, Lorentz-invariant trace

$$
\operatorname{tr} \phi=\phi(0) \Longleftrightarrow \operatorname{tr}(\phi \cdot \psi)=\sum_{s=0}^{\infty} \frac{(-1)^{s}}{s+1} \phi^{\alpha(s) \dot{\alpha}(s)} \psi_{\alpha(s) \dot{\alpha}(s)}
$$

for arbitrary analytic functions $\phi(q)$ and $\psi(q)$. Upon introducing the second-order differential operators

$$
\mathcal{Q}=q^{\alpha \dot{\alpha}} \mathcal{D}_{\alpha} \overline{\mathcal{D}}_{\dot{\alpha}}, \quad \overline{\mathcal{Q}}=-q^{\alpha \dot{\alpha}} \overline{\mathcal{D}}_{\dot{\alpha}} \mathcal{D}_{\alpha}
$$

one finds the identity

$$
\operatorname{tr} \int \mathrm{d}^{8} z E^{-1} \phi \mathcal{Q} \psi=\operatorname{tr} \int \mathrm{d}^{8} z E^{-1}(\overline{\mathcal{Q}} \phi) \psi
$$


is valid for arbitrary superfields $\phi(z, q)$ and $\psi(z, q)$.

Let us consider a linearized theory constructed from an unconstrained complex superfield $U(z, q)$ and real superfields $X(z, q), Y(z, q)$ which all appear in the action functional

$$
\begin{aligned}
S=\frac{1}{2} \operatorname{tr} \int \mathrm{d}^{8} z E^{-1}\{ & \frac{1}{4} Y(\overline{\mathcal{Q}} \mathcal{Q}+4(\overline{\mathcal{P}}-1) \mu \bar{\mu}) X-\frac{\mu \bar{\mu}}{2} Y(\overline{\mathcal{P}}-1)^{2} Y \\
& -\frac{\mu \bar{\mu}}{2} X^{2}-\frac{1}{2} Y(\mathcal{Q} \overline{\mathcal{P}} U+\overline{\mathcal{Q}} \mathcal{P} \bar{U})+\frac{1}{2} X(\mathcal{Q} U+\overline{\mathcal{Q}} \bar{U}) \\
& -\bar{U}(\mathcal{P}+\overline{\mathcal{P}}-2) U-U \overline{\mathcal{P}} U-\bar{U} \mathcal{P} \bar{U}\} .
\end{aligned}
$$

This action remains unchanged under the following gauge transformations

$$
\begin{array}{rlrl}
\delta U & =-\frac{1}{2} \overline{\mathcal{Q}} \bar{\varepsilon}, & \delta \bar{U}=-\frac{1}{2} \mathcal{Q} \varepsilon, \\
\delta Y & =\varepsilon+\bar{\varepsilon}, \quad \delta X=(\overline{\mathcal{P}}-1) \varepsilon+(\mathcal{P}-1) \bar{\varepsilon},
\end{array}
$$

expressed in terms of an unconstrained complex gauge parameter $\varepsilon(z, q)$. The corresponding generators are obviously seen to be linearly independent.

The actions (2.14) and (3.7) can be used to define one and the same physical theory! This can be proven by making use of the following decompositions

$$
\begin{array}{ll}
U=\sum_{s=0}^{\infty} U_{\alpha(s) \dot{\alpha}(s)} q^{\alpha(s) \dot{\alpha}(s)}, \quad U(s, s)=\Gamma(s, s)+G(s, s) \\
Y=\sum_{s=0}^{\infty} Y_{\alpha(s) \dot{\alpha}(s)} q^{\alpha(s) \dot{\alpha}(s)}, \quad Y(s, s)=H^{\prime}(s, s)-H(s, s) \\
X=\sum_{s=0}^{\infty} X_{\alpha(s) \dot{\alpha}(s)} q^{\alpha(s) \dot{\alpha}(s)}, \quad X(s, s)=(s+1)\left\{H^{\prime}(s, s)+H(s, s)\right\}
\end{array}
$$

where (2.20) has been applied to (3.9). Now, calculating the trace in (3.7) reduces it to that given by (2.14). Similarly, representing the gauge parameter in the form

$$
\varepsilon=\sum_{s=0}^{\infty} \varepsilon_{\alpha(s) \dot{\alpha}(s)} q^{\alpha(s) \dot{\alpha}(s)} \quad, \quad \varepsilon(s, s)=\gamma(s, s)-g(s, s)
$$

equation (3.8) proves to be equivalent to the gauge transformations $(2.12),(2.13)$ and (2.19).

As can be seen from (3.10) and (3.11), the expansions of the real $q$-analytic superfields $X$ and $Y$ are expressed in terms of the coefficients $H^{\prime}(s, s)$ and $H(s, s)$. This in turns implies that we may define two additional $q$-analytic superfields via the equations

$$
\begin{aligned}
\mathcal{A}^{\prime} & =\sum_{s=0}^{\infty} H^{\prime}{ }_{\alpha(s) \dot{\alpha}(s)} q^{\alpha(s) \dot{\alpha}(s)} \\
\mathcal{A} & =\sum_{s=0}^{\infty} H_{\alpha(s) \dot{\alpha}(s)} q^{\alpha(s) \dot{\alpha}(s)} .
\end{aligned}
$$


Consequently, we find the results

$$
Y \equiv \mathcal{A}^{\prime}-\mathcal{A} \quad, \quad X \equiv\left[1+q^{\alpha \dot{\alpha}} \frac{\partial}{\partial q^{\alpha \dot{\alpha}}}\right]\left[\mathcal{A}^{\prime}+\mathcal{A}\right]
$$

A remarkable feature of the theory in field is that it admits the simple gauge condition

$$
Y=0 \quad \Rightarrow \quad \varepsilon=i \rho \quad, \quad \rho=\bar{\rho} .
$$

and in this gauge the action reduces to the form

$$
\begin{aligned}
S=\frac{1}{2} \operatorname{tr} \int \mathrm{d}^{8} z E^{-1}\{ & -\frac{\mu \bar{\mu}}{2} X^{2}+\frac{1}{2} X(\mathcal{Q} U+\overline{\mathcal{Q}} \bar{U}) \\
& -\bar{U}(\mathcal{P}+\overline{\mathcal{P}}-2) U-U \overline{\mathcal{P}} U-\bar{U} \mathcal{P} \bar{U}\}
\end{aligned}
$$

and the residual gauge transformations takes the forms

$$
\begin{aligned}
\delta X & =i(\overline{\mathcal{P}}-\mathcal{P}) \rho=-\frac{i}{2 \mu \bar{\mu}}[\mathcal{Q}, \overline{\mathcal{Q}}] \rho, \\
\delta U & =\frac{i}{2} \overline{\mathcal{Q}} \rho, \quad \delta \bar{U}=-\frac{i}{2} \mathcal{Q} \rho,
\end{aligned}
$$

with $\rho$ being a real unconstrained superfield. Here we have used the identity

$$
[\mathcal{Q}, \overline{\mathcal{Q}}]=2 \mu \bar{\mu}(\mathcal{P}-\overline{\mathcal{P}})
$$

An additional surprise is that $X$ is nothing more but an auxiliary superfield. In the gauge (3.16) this can be eliminated with the aid of the equation of motion

$$
X=-\frac{1}{\mu \bar{\mu}}(\mathcal{Q} U+\overline{\mathcal{Q}} \bar{U})
$$

As a result, the theory is completely described by the complex unconstrained superfield $U(z, q)$.

It worth pointing out that any complex superfield has a unique decomposition into the sum of transversal and longitudinal linear ones (see (3.9) and (3.12) ) only in the AdS superspace at $\mu \neq 0$. Nevertheless the action (3.17) has a well defined flat limit. To see this, let us set $\mu=\bar{\mu}$ and make the replacements

$$
U \Rightarrow \sqrt{\mu} U, \quad X \Rightarrow X / \sqrt{\mu}, \quad \rho \Rightarrow \sqrt{\mu} \rho
$$

in (3.17) and (3.22). Due to these re-definitions all the singularities at $\mu=0$ disappear and in this limit we arrive at the action

$$
S=\frac{1}{4} \operatorname{tr} \int \mathrm{d}^{8} z\left\{X(Q U+\bar{Q} \bar{U})-\bar{U}\left(D^{2}+\bar{D}^{2}\right) U-U \bar{D}^{2} U-\bar{U} D^{2} \bar{U}\right\}
$$


and the gauge transformations

$$
\delta X=\frac{i}{2}\left(\bar{D}^{2}-D^{2}\right) \rho \quad, \quad \delta U=\frac{i}{2} \bar{Q} \rho, \quad \delta \bar{U}=-\frac{i}{2} Q \rho,
$$

(here $Q=q^{\alpha \dot{\alpha}} D_{\alpha} \bar{D}_{\dot{\alpha}}$ ) in flat superspace. Note that the superfield $X$ is no longer auxiliary. Although (3.21) is the formal Minkowski space limit of our anti-de-Sitter theory, it will require additional investigation before its full relevance is understood.

It can be seen from (3.10) that the condition $Y=0$ is equivalent to the condition $H=H^{\prime}$. We may ask what is the significance of this? From (2.8) and (2.9), it should be clear that in the gauge $Y=0$, the physical content of both the superspin-s and superspin- $(\mathrm{s}+1 / 2)$ multiplets are realized as component fields in a single real superfield $H$. In the superspace supergravity theory [10 associated with the $N=1$, $D=4$ heterotic string a similar phenomenon is known to occur. In the special $\beta \mathrm{FFC}$ supergeometry of [10], the graviton supermultiplet as well as axion supermultiplet all occur as components of the usual axial-vector supergravity prepotential $H_{\alpha \dot{\alpha}}$. The special $\beta \mathrm{FFC}$ supergeometry is also known in the literature as "the string gauge." Thus, we conjecture that here the condition $Y=0$ may be the analog of the string gauge. It should also be clear that the q-analytic superfield $\mathcal{A}(z, q)$ plays a role analogous to the string-field functional in linearized covariant string field theory.

\section{Global Symmetries}

It follows from (3.22) that the parameters of global symmetries of the unified model should solve of the equation

$$
\mathcal{Q} \Upsilon=0, \quad=\bar{\Upsilon}, \quad \Rightarrow \quad(\mathcal{P}-\overline{\mathcal{P}}) \Upsilon=q^{\alpha \dot{\alpha}} \mathcal{D}_{\alpha \dot{\alpha}} \Upsilon=0
$$

This proves to have the following general solution

$$
\Upsilon=\sum_{s=0}^{\infty}\left\{t_{\alpha(s) \dot{\alpha}(s)}+l_{\alpha(s) \dot{\alpha}(s)}\right\} q^{\alpha(s) \dot{\alpha}(s)}
$$

where the transversal t's and longitudinal l's coefficients are constrained by

$$
t_{\alpha(s) \dot{\alpha}(s)}=\bar{t}_{\alpha(s) \dot{\alpha}(s)}, \quad \overline{\mathcal{D}}^{\dot{\alpha}} t_{\alpha(s) \dot{\alpha}(s)}=\mathcal{D}_{\alpha} \overline{\mathcal{D}}_{\dot{\alpha}} t_{\alpha(s) \dot{\alpha}(s)}=0
$$

and

$$
l_{\alpha(s) \dot{\alpha}(s)}=\bar{l}_{\alpha(s) \dot{\alpha}(s)}, \quad \overline{\mathcal{D}}_{\dot{\alpha}} l_{\alpha(s) \dot{\alpha}(s)}=\mathcal{D}^{\alpha} \overline{\mathcal{D}}^{\dot{\alpha}} l_{\alpha(s) \dot{\alpha}(s)}=0
$$

respectively. The constraints in (4.3) and (4.4) coincide with Killing equations for tensor superfields and define a complete set of independent Killing tensor superfields. In the AdS superspace their solutions are parametrized by a finite number of constant parameters. The component content of the Killing superfields $t(s, s)$ and $l(s, s)$ is given by the Killing tensor fields

$$
\begin{aligned}
& \varepsilon_{\alpha(s) \dot{\alpha}(s)}=t_{\alpha(s) \dot{\alpha}(s)}\left|\quad, \quad \varepsilon_{\alpha(s+1) \dot{\alpha}(s)}=\mathcal{D}_{\alpha} t_{\alpha(s) \dot{\alpha}(s)}\right| \quad, \\
& \varepsilon_{\alpha(s) \dot{\alpha}(s)}=l_{\alpha(s) \dot{\alpha}(s)}\left|\quad, \quad \varepsilon_{\alpha(s-1) \dot{\alpha}(s)}=\mathcal{D}^{\alpha} l_{\alpha(s) \dot{\alpha}(s)}\right|,
\end{aligned}
$$


satisfying the conditions

$$
\nabla^{\alpha \dot{\alpha}} \varepsilon_{\alpha(k) \dot{\alpha}(l)}=0 \quad, \quad \nabla_{\alpha \dot{\alpha}} \varepsilon_{\alpha(k) \dot{\alpha}(l)}=0 \quad .
$$

Here the symbol ' $\mid$ ' indicates the zeroth-order component in $\theta=0, \nabla$ 's denote the covariant derivatives of the AdS space.

The space of Killing tensor superfields (4.3), (4.4) may be endowed with the structure of a superalgebra. Given two tensors, for example both transversal $t(s, s)$ and $t(r, r)$, one can build a Killing longitudinal tensor $l(s+r-2 p+1, s+r-2 p+1)$ for $p=0,1, \ldots, \min (s, r)$ as their antisymmetric bilinear combination defined uniquely modulo a factor from the equations (4.3). In general the following combinations are available

$$
\begin{aligned}
& l(s, s) \text { and } l(r, r) \text { combine to } l(s+r-2 p-1, s+r-2 p-1), \\
& p=0,1, \ldots, \min (s-1, r-1), \\
& t(s, s) \text { and } t(r, r) \text { combine to } l(s+r-2 p+1, s+r-2 p+1) \text {, } \\
& p=0,1, \ldots, \min (s, r) \text {, } \\
& l(s, s) \text { and } t(r, r) \text { combine to } t(s+r-2 p-1, s+r-2 p-1) \text {, } \\
& p=0,1, \ldots, \min (s-1, r) \text {. }
\end{aligned}
$$

Here we present manifestly only the first sector. For that purpose let us introduce the notations $l^{n}(s+n, s-n), n=-s,-s+1, \ldots, s$, and $l^{n+1 / 2}(s+n, s-n-1)$, $n=-s,-s+1, \ldots, s-1,\left(l^{\nu}\right)^{*}=l^{-\nu}$ for the derivatives of the longitudinal Killing tensor $l^{0}(s, s) \equiv l(s, s)$ by the recurrent relations

$$
\begin{gathered}
\mathcal{D}_{\alpha}{ }^{\dot{\alpha}} l_{\alpha(s+n) \dot{\alpha}(s-n)}^{n}=i(s-n+1) \mu l_{\alpha(s+n+1) \dot{\alpha}(s-n-1)}^{n+1}, \\
\overline{\mathcal{D}}^{\dot{\alpha}} l_{\alpha(s+n) \dot{\alpha}(s-n)}^{n}=(s-n+1) \mu l_{\alpha(s+n) \dot{\alpha}(s-n-1)}^{n+1 / 2}, \\
\overline{\mathcal{D}}_{\dot{\alpha}} l_{\alpha(s+n) \dot{\alpha}(s-n-1)}^{n+1 / 2}=-2 l_{\alpha(s+n) \dot{\alpha}(s-n)}^{n},
\end{gathered}
$$

and their complex conjugates. Now if $l(s, s)$ and $l^{\prime}(r, r)$ are two longitudinal Killing superfields, any longitudinal Killing superfield with $s+r-2 p-1$ undotted and dotted indices $(p=0,1, \ldots, \min (s-1, r-1))$ that can be built of $l$ and $l^{\prime}$ is proportional to

$$
\begin{aligned}
& l_{\alpha(s+r-2 p-1) \dot{\alpha}(s+r-2 p-1)}^{\prime \prime}= \\
& \quad \sum_{q} \beta_{q+1 / 2}\left(\sum_{n=-p}^{p} \alpha_{n}^{q+1 / 2} l_{\alpha(s-p+q) \dot{\alpha}(s-p-q-1)}^{n+q+1 / 2 \beta(p+n) \dot{\beta}(p-n)} l_{\alpha(r-p-q-1) \beta(p+n) \dot{\alpha}(r-p+q) \dot{\beta}(p-n)}^{n-q-1 / 2}\right. \\
& \left.\quad+\sum_{n=-p-1}^{p} \alpha_{n+1 / 2}^{q+1 / 2} l_{\alpha(s-p+q) \dot{\alpha}(s-p-q-1)}^{n+q+1 \beta(p+n+1) \dot{\beta}(p-n)} l_{\alpha(r-p-q-1) \beta(p+n+1) \dot{\alpha}(r-p+q) \dot{\beta}(p-n)}^{\prime n-q}\right)(4.1
\end{aligned}
$$

where the summation over $q$ under the conditions $s-p-1 \geq q \geq p-s$ and $r-p-1 \geq$ $q \geq p-r$ and the constants $\beta$ and $\alpha$ are equal

$$
\alpha_{n}^{q+1 / 2}=\frac{i(-1)^{n}}{(p+n) !(p-n) !} \frac{\mu^{n}}{\bar{\mu}^{n}} \quad, \quad \alpha_{n+1 / 2}^{q+1 / 2}=\frac{2 i(-1)^{n+1}}{(p+n+1) !(p-n) !} \frac{\mu^{n}}{\bar{\mu}^{n+1}}
$$




$$
\beta_{q+1 / 2}=[(s-p+q) !(s-p-q-1) !(r-p+q) !(r-p-q-1) !]^{-1} .
$$

They satisfy the relations

$$
\bar{\beta}_{q+1 / 2}=\beta_{-q-1 / 2} \quad, \quad \bar{\alpha}_{n}^{q+1 / 2}=-\alpha_{-n}^{-q-1 / 2}, \quad \bar{\alpha}_{n+1 / 2}^{q+1 / 2}=\alpha_{-n-1 / 2}^{-q-1 / 2},
$$

that ensure simultaneously the reality of $l^{\prime \prime}$ and its antisymmetricity with respect to the replacement $l(s, s) \leftrightarrow l^{\prime}(r, r)$.

Let us consider the lowest components of the Killing superfield $\Upsilon$, namely $t$ and $l_{\alpha \dot{\alpha}}$. They prove to describe important symmetries of the model: $N=1$ AdS transformations (see, e.g., [2, 11]) and $N=2$ global supersymmetry [12 respectively.

The $N=1$ AdS transformations can be written in the form

$$
\delta X=\mathcal{K} X, \quad \delta Y=\mathcal{K} Y, \quad \delta U=\mathcal{K} U
$$

where the operator $\mathcal{K}$ is expressed in terms of special Killing superfield $\Upsilon$ :

$$
\mathcal{K}=\frac{1}{2} q^{\alpha \dot{\alpha}}\left\{\frac{i}{2}\left(\mathcal{D}_{\alpha} \Upsilon\right) \overline{\mathcal{D}}_{\dot{\alpha}}+\text { c.c. }+\left(M_{\alpha}{ }^{\beta} \Upsilon\right) \mathcal{D}_{\beta \dot{\alpha}}-\left(\mathcal{D}_{\beta \dot{\alpha}} \Upsilon\right) M^{\beta}{ }_{\alpha}+\frac{1}{2} \Upsilon \mathcal{D}_{\alpha \dot{\alpha}}\right\}, .
$$

where $\Upsilon \equiv q^{\alpha \dot{\alpha}} k_{\alpha \dot{\alpha}}$. Another symmetry of the action (3.17) involves the scalar transversal component $t$ of the Killing superfield $\Upsilon$ :

$$
\begin{aligned}
\delta_{t} Y= & 0 \quad, \quad \delta_{t} X=2 i[\overline{\mathcal{P}}, t] \mathcal{R} U+\text { c.c. } \quad, \\
\delta_{t} U= & \frac{i}{2} \mu \bar{\mu}[\mathcal{P}+\overline{\mathcal{P}}, t] \mathcal{R} X+\frac{i}{2} \mu \bar{\mu}(\overline{\mathcal{P}}-1)[\mathcal{P}+\overline{\mathcal{P}}, t] \mathcal{R} Y \\
& +i q^{\alpha \dot{\alpha}}\left(\mathcal{D}_{\alpha} t\right) \overline{\mathcal{D}}_{\dot{\alpha}} \mathcal{R}(U+\bar{U})
\end{aligned}
$$

where $R$ denotes the reflection on the hyperboloid

$$
\mathcal{R} \phi(q)=\phi(-q)
$$

These are exactly the $N=2$ supersymmetry transformations and $O(2)$ rotations of the $N=2$ AdS superalgebra, which were found in components in 12. Therefore we conclude that the action (3.17) possesses $N=2$ AdS supersymmetry which is described in the covariant form in terms of the generating superfields $X(z, q), Y(z, q)$ and $U(z, q)$ with the Killing parameter $\Upsilon(z, q)$ containing only $t$ and $l_{\alpha \dot{\alpha}}$ components. The $N=2$ transformations turn out to form a closed algebra off the mass-shell. In the gauge $Y=0$ a commutator of two transformations (4.13) is given by the sum of an AdS transformation (4.11) and a gauge variation:

$$
\begin{aligned}
{\left[\delta_{t}, \delta_{t^{\prime}}\right] X } & =\mathcal{K} X+i(\overline{\mathcal{P}}-\mathcal{P}) \rho \\
{\left[\delta_{t}, \delta_{t^{\prime}}\right] U } & =\mathcal{K} U+\frac{i}{2} \overline{\mathcal{Q}} \rho
\end{aligned}
$$

where $\mathcal{K}$ is given by (4.11) with

$$
\Upsilon=4 i\left(\left(\mathcal{D}_{\alpha} t\right)\left(\overline{\mathcal{D}}_{\dot{\alpha}} t^{\prime}\right)-\left(\mathcal{D}_{\alpha} t\right)\left(\overline{\mathcal{D}}_{\dot{\alpha}} t^{\prime}\right)\right) q^{\alpha \dot{\alpha}}, \quad \rho=\frac{1}{2} \Upsilon(U+\bar{U})
$$


In the work of [7] the superalgebra of higher spins and auxiliary fields shsa(1) was proposed to play the role of the structure algebra of a higher spin theory. This algebra describes the global symmetries of the free action as well. As the spin content of our model coincides with that arising in the framework of [4], the superalgebra shsa(1) can be considered as a global symmetry algebra of the action (3.17) too. It is then natural to identify the $N=2$ AdS superalgebra of the transformations (4.11) and (4.13) with the finite-dimensional subalgebra of $\operatorname{shsa}(1)$. The fact that these transformations are expressed in terms of Killing superfields along with some observations at the component level allows us to suggest that all the global symmetries can be expressed in terms of Killing tensors (4.3) and (4.4). Then the (anti)commutator relations of the superalgebra of global symmetries could be described by the bilinear combinations (4.6), (4.10) after appropriate normalization of the multiplicative factors. Most likely the Killing tensors entering the superfield $\Upsilon$ (4.10) correspond only to the physical subalgebra of shsa(1) (at equal powers of the Klein operators [7]) because the number and the Lorentz structure of generators in this subalgebra coincides with those of the constant parameters in the solutions of (4.3) and (4.4).

In closing this work, we also note that the issue of duality can also be investigated within the context of the universal action in (3.7) as there seems to be no fundamental impediment to performing a superfield duality transformation upon this action. Presumably such a duality transformation would replace the chiral scalar multiplet described in (2.15) and (2.16) by a chiral spinor superfield since the latter is known to describe the axion multiplet in the work on the $N=1, D=4$ supergeometry [10] that arises from the heterotic string. It would be an interesting exercise to show that the duality transformation when implemented on the generating formalism, does indeed interchange the two dually equivalent superfield formulations [3]. But then it is worth expecting the appearance, in the generating formalism, of inverse powers of $(2 \mathcal{P}-1)$ which turn into numerical factors only upon passing to the components in $q$ and decomposing the complex unconstrained superfields into their transversal and longitudinal parts by the rule (2.20).

\section{Acknowledgements}

One of us (SMK) thanks the Institute for Theoretical Physics at Hannover, where the present paper was completed, for hospitality. This work was supported in part by the Russain Foundation for Basic Research Grant No. 96-02-16017 and the Joint DFG-RFBR Project Grant No. 96-02-00180G. The research of SJG was supported by the U.S. NSF under grant PHY-96-43219 and NATO under grant CRG-930789. The work of AGS was also supported by a Fellowship of Tomalla Foundation (under the program of the Interanational Center for Fundamental Physics in Moscow). 


\section{References}

[1] S.M. Kuzenko, V.V. Postnikov and A.G. Sibiryakov, JETP Lett. 57 (1993) 534;

S.M. Kuzenko and A.G. Sibiryakov, JETP Lett. 57 (1993) 539.

[2] I.L. Buchbinder and S.M. Kuzenko, Ideas and Methods of Supersymmetry and Supergravity. Institute of Physics Publishing, Bristol and Philadelphia, 1995.

[3] S.M. Kuzenko and A.G. Sibiryakov, Phys. At. Nucl. 57 (1994) 1257.

[4] M.A. Vasiliev, Phys. Lett. B243 (1990) 378; Class. Quantum Grav. 8 (1991) 1387; Phys. Lett. B285 (1992) 225.

[5] C. Fronsdal, Phys. Rev. D20 (1979) 848; J. Fang and C. Fronsdal, Phys. Rev. D22 (1980) 1361.

[6] M.A. Vasiliev, Fortschr. Phys. 35 (1987) 741.

[7] E.S. Fradkin and M.A. Vasiliev, Int. J. Mod. Phys. A3 (1988) 2983.

[8] B.W. Keck, J. Phys. A8 (1975) 1819; B. Zumino, Nucl. Phys. B127 (1977) 189; E.A. Ivanov and A.S. Sorin, J. Phys. A13 (1980) 1159.

[9] J. Wess and J. Bagger, Supersymmetry and Supergravity. Cambridge Univ. Press, Cambridge, 1983.

[10] S.J. Gates, P. Majumdar, R. Oerter and A. E. van de Ven, Phys. Lett. 214B, (1988) 26; idem. Nucl. Phys. B319 (1989) 291.

[11] S.J. Gates, M.T. Grisaru, M. Roček and W. Siegel, Superspace. BenjaminCummings, Reading, MA, 1983.

[12] S.J. Gates, S.M. Kuzenko and A.G. Sibiryakov, $N=2$ Supersymmetry of Higher Superspin Massless Theories. preprint UMDEPP 97-23, TSU-QFT-13/96; hepth/9609141, to appear in Phys. Lett. B. 